\title{
STUDY OF MAIN BIFURCATION SCENARIOS FOR FUNCTIONAL DIFFERENTIAL TIME-DELAY EQUATIONS
}

\author{
M.G. YUMAGULOV, D.A. YAKSHIBAEVA
}

\begin{abstract}
In the paper we study the main bifurcation scenarios for functional differential time delay equations with periodic right side and for nonlinear autonomous equations with aftereffect. The main tool is the operator method for studying multi-parameter bifurcation leading us to new sufficient bifurcation conditions and allowing us to obtain the approximate formulae for appearing solutions. As applications, we consider the problems on bifurcation points for the modifications of Duffing equation and Hutchinson-Wright equation.
\end{abstract}

Keywords: functional differential equations, time-delay system, dynamical systems, bifurcation, operator method, functionalization parameter, asymptotic formula

Mathematics Subject Classification: 34C23, 34K05, 34K13, 34K18

\section{Formulation of PROBlem}

In the paper we consider systems of functional differential time-delay equations

$$
\frac{d x(t)}{d t}=\int_{0}^{\sigma}\left[d_{\tau} R(t, \tau)\right] x(t-\tau)+\int_{0}^{\sigma}\left[d_{\tau} Q(t, \tau)\right] \Phi[x(t-\tau)]+F\left(t, x_{t}\right),
$$

where $x \in \mathbb{R}^{N}$. Here $\sigma>0, R(t, \tau), Q(t, \tau)$ are $N \times N$ matrices whose entries are defined for $-\infty<t<\infty, 0 \leqslant \tau \leqslant \sigma$, and they are functions of bounded variations w.r.t. $\tau$ are continuous in w.r.t. $t$ in the following sense: for each $t$, the identities

$$
\lim _{t^{\prime} \rightarrow t} \int_{0}^{\sigma}\left\|R\left(t^{\prime}, \tau\right)-R(t, \tau)\right\| d \tau=0, \lim _{t^{\prime} \rightarrow t} \int_{0}^{\sigma}\left\|Q\left(t^{\prime}, \tau\right)-Q(t, \tau)\right\| d \tau=0
$$

hold true, and functions $R(t, \tau)$ and $Q(t, \tau)$ are $T$-periodic in $t: R(t+T, \tau)=R(t, \tau)$, $Q(t+T, \tau)=Q(t, \tau)$. In (1) we have employed the notation $x_{t}=\left(x\left(t-\phi_{1}\right), \ldots, x\left(t-\phi_{s}\right)\right)$, $0 \leqslant \phi_{1}<\phi_{2}<\ldots<\phi_{s} \leqslant \sigma, s$ is a natural number. The nonlinearity $F\left(t, x_{t}\right)$ is not represented by an integral $\int_{0}^{\sigma}\left[d_{\tau} A(t, \tau)\right] \Psi[x(t-\tau)]$. Let $y=\left(y_{1}, y_{2}, \ldots, y_{s}\right)$; it is assumed that vector functions $\Phi(x), F(t, y)$ are continuously differentiable and satisfy the conditions

$$
\|\Phi(x)\|=O\left(\|x\|^{2}\right),\|x\| \rightarrow 0,\|F(t, y)\|=O\left(\|y\|^{2}\right),\|y\| \rightarrow 0
$$

uniformly in $t, F(t+T, y)=F(t, y)$. Hereinafter the symbol $\|\cdot\|$ denotes the Euclidean norm of vector and matrices in spaces $\mathbb{R}^{N}$ or $\mathbb{R}^{s}$. The integrals in (1) are treated in the LebesgueStieltjes sense.

M.G. Yumagulov, D.A. Yakshibaeva, Study of main Bifurcation scenarios for functional DIFFERENTIAL TIME-DELAY EQUATIONS.

(c) Yumagulov M.G., Yakshibaeva D.A. 2014.

The work is supported by the Ministery of Education and Science of Russian Federation (agreement 14.37.21.0358) and partially by RFBR (grants 11-01-97009 and 12-01-00567-a).

Submitted November 25, 2013. 
Many interesting equations with time-delay can be reduced to equation (1) (see, for instance, [1]-[3]). In particular, if $R(t, \tau), Q(t, \tau)$, and $F\left(t, x_{t}\right)$ are independent of $t$, we obtain the autonomous equation

$$
\frac{d x(t)}{d t}=\int_{0}^{\sigma}\left[d_{\tau} R(\tau)\right] x(t-\tau)+\int_{0}^{\sigma}\left[d_{\tau} Q(\tau)\right] \Phi[x(t-\tau)]+F\left(x_{t}\right) .
$$

Systems (1) and (2) have a solution $x \equiv 0$. An important feature of this solution is the hyperbolicity property. Let us give appropriate definitions.

First we consider equation (1). We denote by $V$ the monodromy matrix of linear system

$$
\frac{d x(t)}{d t}=\int_{0}^{\sigma}\left[d_{\tau} R(t, \tau)\right] x(t-\tau) .
$$

The solution $x=0$ to system (1) is called (cf., for instance, [3]) hyperbolic equilibrium if matrix $V$ has no eigenvalues with modulus 1 . Otherwise $x=0$ is called non-hyperbolic equilibrium of system (1).

Consider autonomous equation (2). We define characteristic quasi-polynomial

$$
L(p)=\operatorname{det}\left(\int_{0}^{\sigma} d_{\tau} R(\tau) e^{-p \tau}-p I\right)
$$

associated with the linear part of equation (2). The solution $x=0$ is called hyperbolic equilibrium of system (2) if quasi-polynomial (3) has no pure imaginary zeroes. Otherwise $x=0 \mathrm{~s}$ called non-hyperbolic equilibrium of system (2).

In applications, systems (1) and (2) depend usually on parameters and variation of these parameters can change hyperbolicity that produces various bifurcations in the vicinity of point $x=0$. A large amount of works is devoted to studying bifurcations in functional differential time-delay equations (see, for instance, [4]-[8]). In these works there were proposed effective approaches for obtaining bifurcation tests, for approximate representation of solutions, and for studying the stability. These approaches employ the Poincaré normal forms method, central manifold theorem, topological methods, etc.

In the present work we study problem on bifurcation of periodic solutions to equations (1) and (2). To solve these problems, we propose a development of an operator approach for studying multi-parametric bifurcations [9]. The mentioned method allows us to establish sufficient bifurcation conditions and to obtain asymptotic formulae for bifurcating solutions. In contrast to the usually applied methods, the proposed algorithm does not involve construction of normal forms and integral manifolds that in many cases allows us to simplify the approximate study of bifurcations and to obtain simple bifurcation tests directly in terms of the original problem.

\section{BIFURCATION SCENARIOS FOR NON-AUTONOMOUS SYSTEMS}

First we consider system (1) depending on a scalar or vector parameter $\theta$ and having a $T$-periodic in $t$ right hand side:

$$
\frac{d x(t)}{d t}=\int_{0}^{\sigma(\theta)}\left[d_{\tau} R(\theta, t, \tau)\right] x(t-\tau)+\int_{0}^{\sigma(\theta)}\left[d_{\tau} Q(\theta, t, \tau)\right] \Phi[\theta, x(t-\tau)]+F\left(\theta, t, x_{t}\right),
$$

where $\sigma(\theta)$ is infinitely differentiable function obeying $0<\sigma(\theta)<T$.

We call a value $\theta=\theta_{0}$ bifurcation point in the vicinity of solution $x=0$ if $x=0$ is a non-hyperbolic equilibrium of equation (4) as $\theta=\theta_{0}$. 
We denote by $V(\theta)$ the monodromy matrix of linearized system (4). In what follows we consider situations when monodromy matrix $V\left(\theta_{0}\right)$ has a simple eigenvalue 1 or a pair of simple eigenvalues $e^{ \pm 2 \pi p i / q}$, where $0 \leqslant \frac{p}{q} \leqslant \frac{1}{2}$ and $\frac{p}{q}$ are rational irreducible fractions. In both case we assume that the moduli of other eigenvalues of matrix $V\left(\theta_{0}\right)$ are not equal to one. Subject to these cases, various scenarios of local bifurcations in the vicinity of equilibrium of system (4) are possible.

2.1. Forced vibrations bifurcations. In the case $V\left(\theta_{0}\right)$ has a simple eigenvalue 1 and the moduli of other eigenvalues are not equal to 1 , the codimension of the bifurcation equals one. In this case it is natural to deal with scalar parameter $\theta$. Here the main bifurcation scenario is the emergence of nonzero $T$-periodic oscillations with small amplitude of system in the vicinity of equilibrium $x=0$ as parameter $\theta$ passes through $\theta_{0}$. This bifurcation corresponds to the following notion.

A value $\theta_{0}$ of parameter $\theta$ is called bifurcation point of forced vibrations of system (4) if for each $\varepsilon>0$ there exists $\theta=\theta(\varepsilon)$ such that system (4) has a nonzero $T$-periodic solution $x(t, \varepsilon)$, and $\theta(\varepsilon) \rightarrow \theta_{0}$ and $\max _{t}\|x(t, \varepsilon)\| \rightarrow 0$ as $\varepsilon \rightarrow 0$.

Let us provide a sufficient condition for forced oscillations bifurcations. In order to do it, given a function $x=x(t)$ on the segment $[0, T]$ and a number $\tau, 0 \leq \tau \leq T$, we define the function $u(t)$ and the operator $E\left(\frac{\tau}{T}\right)$ by the identities

$$
u(t)=x(T \cdot t) \text { and } E\left(\frac{\tau}{T}\right) u(s)= \begin{cases}u\left(s-\frac{\tau}{T}+1\right), & 0 \leqslant s<\frac{\tau}{T}, \\ u\left(s-\frac{\tau}{T}\right), & \frac{\tau}{T} \leqslant s \leqslant 1 .\end{cases}
$$

We introduce the operator

$$
B(\theta) u(t)=u(1)+T \int_{0}^{t}\left(\int_{0}^{\sigma(\theta)} d_{\tau} R(\theta, T s, \tau) E\left(\frac{\tau}{T}\right) u(s)\right) d s
$$

acting and being continuous in the space $L_{2}[0,1]$ on a dense domain $C[0,1]$.

Lemma 1. If $V\left(\theta_{0}\right)$ has a simple eigenvalue 1 , operator $B\left(\theta_{0}\right): L_{2} \rightarrow L_{2}$ has a simple eigenvalue 1.

The lemma can be proved by simple calculations.

Let $e(t)$ be an eigenfunction of operator $B\left(\theta_{0}\right)$ associated with the eigenvalue 1 . The adjoint operator $B^{*}\left(\theta_{0}\right): L_{2} \rightarrow L_{2}$ has also an eigenvalue 1 and the associated eigenfunction is $e^{*}(t)$. We choose functions $e(t)$ and $e^{*}(t)$ by the restriction $\left(e, e^{*}\right) \neq 0$; hereinafter $(\cdot, \cdot)$ denotes the scalar product in $L_{2}$.

Theorem 1. Let $V\left(\theta_{0}\right)$ have a simple eigenvalue 1 and the relation

$$
\left(B_{\theta}^{\prime}\left(\theta_{0}\right) e, e^{*}\right) \neq 0
$$

is satisfied. Then value $\theta_{0}$ of parameter $\theta$ is a bifurcation point of forced oscillation for equation (4). Here $B_{\theta}^{\prime}$ is the operator obtained by differentiation of operator $B(\theta)$ w.r.t. $\theta$.

The proofs for this and other statements of the work are given in Section 4.

2.2. Subharmonic oscillation bifurcation. Here we consider the case when the monodromy matrix $V\left(\theta_{0}\right)$ has two simple eigenvalues $e^{ \pm 2 \pi i \frac{p}{q}}$, where $0 \leqslant \frac{p}{q} \leqslant \frac{1}{2}$ are $\frac{p}{q}$ rational irreducible fractions and the moduli of other eigenvalues of matrix $V\left(\theta_{0}\right)$ are not equal to one. In this case the codimension of the bifurcation is two. Here it is natural to assume that parameter $\theta$ is two-dimensional, i.e., $\theta=(\alpha, \beta)$, where $\alpha$ and $\beta$ are scalar parameters. We let 
$\theta_{0}=\left(\alpha_{0}, \beta_{0}\right)$. The main bifurcation scenario is the emergence of $q T$-periodic solutions in the vicinity of the equilibrium $x=0$ as parameter $\theta$ passes through $\theta_{0}$.

A value $\theta_{0}$ of parameter $\theta$ is called bifurcation point of $q T$-periodic subharmonic oscillations for system (4) if for each $\varepsilon>0$ there exists $\theta=\theta(\varepsilon)$ such that system (4) has a non-zero $q T$-periodic solution $x(t, \varepsilon)$, at that, $\theta(\varepsilon) \rightarrow \theta_{0}$ and $\max _{t}\|x(t, \varepsilon)\| \rightarrow 0$ as $\varepsilon \rightarrow 0$.

We define operator $B(\alpha, \beta)$ by analogy with (5), where value $T$ should be replaced by $q T$.

Lemma 2. Suppose that monodromy matrix $V\left(\theta_{0}\right)$ has a pair of simple eigenvalues $e^{ \pm 2 \pi i \frac{p}{q}}$, where $0 \leqslant \frac{p}{q} \leqslant \frac{1}{2}$ and $\frac{p}{q}$ are rational irreducible fractions. At that, let matrix $V\left(\theta_{0}\right)$ has no other eigenvalues with modulus 1 . Then operator $B\left(\alpha_{0}, \beta_{0}\right): L_{2} \rightarrow L_{2}$ has a semi-simple eigenvalue 1 of multiplicity two.

We denote by $e=e(t)$ and $g=g(t)$ linearly independent eigenfunctions of operator $B_{0}=$ $B\left(\alpha_{0}, \beta_{0}\right): B_{0} e=e, B_{0} g=g$. The adjoint operator $B_{0}^{*}$ has also a semi-simple eigenvalue 1 of multiplicity two and the associated eigenfunctions are $e^{*}=e^{*}(t)$ and $g^{*}=g^{*}(t)$. We choose these eigenfunctions by the restrictions

$$
\left(e, e^{*}\right)=\left(g, g^{*}\right) \neq 0, \quad\left(e, g^{*}\right)=\left(g, e^{*}\right)=0 .
$$

Theorem 2. Assume the hypothesis of Lemma 2 and

$$
\Delta=\operatorname{det}\left[\begin{array}{ll}
\left(B_{\alpha}^{\prime}\left(\alpha_{0}, \beta_{0}\right) e, e^{*}\right) & \left(B_{\beta}^{\prime}\left(\alpha_{0}, \beta_{0}\right) e, e^{*}\right) \\
\left(B_{\alpha}^{\prime}\left(\alpha_{0}, \beta_{0}\right) e, g^{*}\right) & \left(B_{\beta}^{\prime}\left(\alpha_{0}, \beta_{0}\right) e, g^{*}\right)
\end{array}\right] \neq 0
$$

Then the pair of number $\left(\alpha_{0}, \beta_{0}\right)$ is the bifurcation point of subharmonic oscillations of system (4). Here $B_{\alpha}^{\prime}$ and $B_{\beta}^{\prime}$ are the operators obtained by differentiation of operator $B(\alpha, \beta)$.

Example 1. Consider the modified Duffing equation

$$
y^{\prime \prime}(t)+\alpha y^{\prime}(t-1)+\left(\frac{1}{4}+\beta \cos t\right) y(t)=-(y(t-2))^{3} \sin t .
$$

Letting $x_{1}=y^{\prime}, x_{2}=y$ and $x=\left(x_{1}, x_{2}\right)^{T}$, we rewrite equation (9) as equivalent system (4), where

$$
\begin{gathered}
R(\alpha, \beta, t, \tau)=\left(\begin{array}{cc}
-\alpha h(\tau-1) & -\left(\frac{1}{4}+\beta \cos t\right) h(\tau) \\
h(\tau) & 0
\end{array}\right), \quad \Phi(\alpha, \beta, x)=x^{3}, \\
Q(\alpha, \beta, t, \tau)=\left(\begin{array}{cc}
0 & -h(\tau-2) \sin t \\
0 & 0
\end{array}\right), \quad F\left(\alpha, \beta, t, x_{t}\right)=0 .
\end{gathered}
$$

Here $h(\tau)$ is the Heaviside function.

Let us study the issue on local bifurcations of the system in the vicinity of equilibrium $x=0$. In our example $T=2 \pi$. As $\alpha_{0}=0$ and $\beta_{0}=0$, the monodromy matrix for linearized equation (9) has a semi-simple eigenvalue -1 of multiplicity two, i.e., it has a pair of simple eigenvalues $e^{ \pm 2 \pi i \frac{p}{q}}$ as $\frac{p}{q}=\frac{1}{2}$. This is why equation (9) satisfies the necessary condition for bifurcation of subharmonic oscillations given in Theorem 2 as $q=2$.

By simple calculations one can make sure that in the considered example determinant (8) is non-zero, namely, $\triangle=-4 \pi^{2}$. Therefore, in accordance with Theorem 2, the pair $\alpha_{0}=0$ and $\beta_{0}=0$ forms a bifurcation point of twice period for equation (9). That is, in the vicinity of zero solution there appear $4 \pi$-periodic solutions. 


\section{BifurCATION SCENARIO FOR AUTONOMOUS SYSTEMS}

Consider autonomous system (2) with a time-delay depending on parameter $\theta$ :

$$
\frac{d x(t)}{d t}=\int_{0}^{\sigma(\theta)}\left[d_{\tau} R(\theta, \tau)\right] x(t-\tau)+\int_{0}^{\sigma(\theta)}\left[d_{\tau} Q(\theta, \tau)\right] \Phi(\theta, x(t-\tau))+F\left(\theta, x_{t}\right),
$$

where $\sigma(\theta)>0$. Parameter $\theta$ is supposed to be scalar.

A value $\theta=\theta_{0}$ is called bifurcation point in the vicinity of solution $x=0$ for equation (10) if characteristic quasi-polynomial $L(p, \theta)$ has pure imaginary zeroes $p= \pm i \omega_{0}$ as $\theta=\theta_{0}$; here $\omega_{0} \geqslant 0$.

We restrict ourselves by considering the most interesting case $\omega_{0}>0$ corresponding to Andronov-Hopf bifurcation. A value $\theta=\theta_{0}$ is called Andronov-Hopf bifurcation point for system (10) if there exists $\theta_{n} \rightarrow \theta$ such that as $\theta=\theta_{n}$, equation (10) has a stationary periodic solution $x_{n}(t)$ and $\max _{t}\left\|x_{n}(t)\right\| \rightarrow 0$ as $n \rightarrow \infty$.

Let us provide a sufficient condition of Andronov-Hopf bifurcation. In order to do it, in the space $L_{2}[0,1]$ with introduce the operator

$$
B(\theta, T) u(t)=u(1)+T \int_{0}^{t}\left(\int_{0}^{\sigma(\theta)} d_{\tau} R(\theta, \tau) E\left(\frac{\tau}{T}\right) u(s)\right) d s
$$

on the dense domain $C[0,1]$.

Lemma 3. Suppose that for some $\omega_{0}>0$ the identity $L\left( \pm i \omega_{0}, \theta_{0}\right)=0$ holds true and $L\left( \pm i m \omega_{0}, \theta_{0}\right) \neq 0, m=0,2,3, \ldots$ Then operator $B\left(\theta_{0}, T_{0}\right)$ has a semi-simple eigenvalue 1 of multiplicity two.

We denote by $e=e(t), g=g(t), e=e^{*}(t)$, and $g=g^{*}(t)$ the eigenfunctions of operators $B\left(\theta_{0}, T_{0}\right), B^{*}\left(\theta_{0}, T_{0}\right)$, respectively. The eigenfunctions are chosen by restrictions (7).

Theorem 3. Suppose the hypothesis of Lemma 3 and the relation

$$
\operatorname{det}\left[\begin{array}{cc}
\left(B_{\theta}^{\prime}\left(T_{0}, \theta_{0}\right) e, e^{*}\right) & \left(B_{T}^{\prime}\left(T_{0}, \theta_{0}\right) e, e^{*}\right) \\
\left(B_{\theta}^{\prime}\left(T_{0}, \theta_{0}\right) e, g^{*}\right) & \left(B_{T}^{\prime}\left(T_{0}, \theta_{0}\right) e, g^{*}\right)
\end{array}\right] \neq 0
$$

where $T_{0}=\frac{2 \pi}{\omega_{0}}$. Then value $\theta_{0}$ of parameter $\theta$ is the Andronov-Hopf bifurcation point for equation (10). Here $B_{\theta}^{\prime}$ and $B_{T}^{\prime}$ are the operators obtained by differentiation of operator $B(\theta, T)$ w.r.t. $\theta$ and $T$, respectively.

Example 2. Consider Hutchinson-Wright equation (cf. [11])

$$
x^{\prime}(t)=-\frac{\pi}{2} x(t-\theta)[1+x(t)], \quad \theta \geqslant 0,
$$

The parameter in this equation is delay $\theta$.

In the considered example we have

$$
\sigma(\theta)=\theta, \quad R(\theta, \tau)=-\frac{\pi}{2} H(\tau-\theta), \quad Q(\theta, \tau)=0, \quad F\left[x(t), x\left(t-\phi_{1}(\theta)\right)\right]=-\frac{\pi}{2} x(t) x(t-\theta) .
$$


The first condition of Theorem implies the system

$$
\left\{\begin{array}{l}
\int_{0}^{\theta_{0}} \cos \left(\frac{2 \pi}{T_{0}} \tau\right) d_{\tau} H\left(\tau-\theta_{0}\right)=0 \\
\int_{0}^{\theta_{0}} \sin \left(\frac{2 \pi}{T_{0}} \tau\right) d_{\tau} H\left(\tau-\theta_{0}\right)=\frac{4}{T_{0}},
\end{array}\right.
$$

having the solution $\theta_{0}=1+4 n, n \geqslant 0, n$ is integer, and $T_{0}=4$.

Suppose, for instance, that $\theta_{0}=1$ and $T_{0}=4$. Then checking relation (12), we arrive at the identity

$$
\operatorname{det}\left[\begin{array}{cc}
0 & \frac{1}{8} \\
-\frac{\pi}{4} & \frac{\pi}{16}
\end{array}\right]=\frac{\pi}{32} \text {. }
$$

Thus, the hypothesis of Theorem 3 is satisfied and hence, the number $\theta_{0}=1$ is an AndronovHopf bifurcation point for equation (13).

\section{Proof of main Statements}

To prove Theorems 1-3 we employ the operator method of studying local bifurcations of operator equations (see [9] and [12]).

Proof of Theorem 1. We pass to the operator equation

$$
u(t)=B(\theta) u(t)+b[\theta, u(t)],
$$

where $B(\theta)$ is operator (5), and $b[\theta, u(t)]$ is the operator

$$
\begin{aligned}
b[\theta, u(t)]= & T \int_{0}^{t}\left(\int_{0}^{\sigma(\theta)}\left[d_{\tau} Q(\theta, T s, \tau)\right] E\left(\frac{\tau}{T}\right) \Phi[\theta, u(s)]\right) d s \\
& +T \int_{0}^{t}\left(F\left(\theta, T s, E\left(\frac{\phi_{1}}{T}\right) u(s), \ldots, E\left(\frac{\phi_{s}}{T}\right) u(s)\right)\right) d s .
\end{aligned}
$$

A simple check shows that $T$-periodic solutions $x(t)$ to equation (4) coincide with solutions $u(t)$ to equation (14).

As the basis of the procedure of constructing bifurcating solutions to equation (14), we choose the parameter functionalization method [13 and the modified Newton-Kantorovich method with perturbations [14].

At the first step we consider functionalized equation

$$
u=B(\theta(u)) u+b[\theta(u), u],
$$

where $\theta(u)$ is a continuous functional, which we choose as

$$
\theta(u)=\frac{\theta_{0}}{\varepsilon}\left(u, e^{*}\right)
$$

where $e^{*}$ is an eigenfunction of operator $B^{*}, \varepsilon>0$ is a small auxiliary parameter. If $u^{*}$ is a solution to equation (16), then $u^{*}$ solves equation (14) as $\theta=\theta\left(u^{*}\right)$.

At the second step we study equation (16) by the Newton-Kantorovich method. In order to do it, we represent $(16)$ as

$$
G(u)+W(u)=0,
$$

where $G(u)=u-B[\theta(u)] u, W(u)=-b[\theta(u), u]$. 
Operators $G$ and $W$ act in the space $L_{2}[0,1]$ and depend on parameter $\varepsilon>0$. For the sake of simplicity, we do not use $\varepsilon$ in the notations for operators. Space $L_{2}$ can be represented as $L_{2}=H_{0} \oplus H^{0}$, where $H_{0}$ is the eigenspace associated with the simple eigenvalue of the operator $B_{0}: B_{0}=B\left(\theta_{0}\right)$, and $H^{0}$ is the additional invariant for $B_{0}$ subspace. We let $G^{\prime}(\varepsilon e) h=h-B\left(\theta_{0}\right) h-\theta_{0}\left(h, e^{*}\right) B^{\prime}\left(\theta_{0}\right) e$ and $u_{0}=\varepsilon e$. It follows from condition (7) that there exists a bounded operator $\Gamma_{0}=\left[G^{\prime}\left(u_{0}\right)\right]^{-1}: L_{2} \rightarrow L_{2}$, at that, operator $\Gamma_{0}$ is independent of $\varepsilon$. Operator $\Gamma_{0}$ can be calculated by the formula $\Gamma_{0} y=h_{0}+h^{0}$, where

$$
h_{0}=-\frac{\left(y, e^{*}\right) e}{\theta_{0}\left(B^{\prime}\left(\theta_{0}\right) e, e^{*}\right)}, \quad h^{0}=\left(I-B_{0}\right)^{-1}\left[y-\frac{\left(y, e^{*}\right) B^{\prime}\left(\theta_{0}\right) e}{\left(B^{\prime}\left(\theta_{0}\right) e, e^{*}\right)}\right] .
$$

Lemma 4. Suppose the hypothesis of Theorem 1. Then, for each small $\varepsilon>0$ equation (17) has a nontrivial solution $u(\varepsilon)$, which can be obtained as the limit of successive approximations,

$$
u_{n+1}=u_{n}-\Gamma_{0} G\left(u_{n}\right)-\Gamma_{0} W\left(u_{n}\right), n=0,1,2, \ldots,
$$

where $u_{0}=u_{0}(t)=\varepsilon e(t)$.

The above constructions imply that as $\theta=\theta[u(\varepsilon)]$, equation (14) has nonzero solutions $u(\varepsilon)$ such that $\|u(\varepsilon)-\varepsilon e\|=o(\varepsilon), \theta[u(\varepsilon)] \rightarrow \theta_{0}$ as $\varepsilon \rightarrow 0$. It completes the proof of Theorem 1 .

We note that Lemma 4 implies the existence the function $\theta(\varepsilon)=\theta[x(\varepsilon)]$ such that as $\theta=\theta(\varepsilon)$, equation (4) has a nontrivial $T$-periodic solution $x(t, \varepsilon): x(0, \varepsilon)=x(\varepsilon)$. And values of functions $\theta(\varepsilon)$ and $x(\varepsilon)$ can be constructed by iterations (18), where $u(t)=x(T \cdot t)$.

Proof of Theorem 2. The problem on bifurcation of subharmonic oscillations for equation (4) is equivalent to the problem on bifurcation of small nontrivial solutions to the operator equation

$$
u(t)=B(\alpha, \beta) u(t)+b(\alpha, \beta, u(t)),
$$

where $B(\alpha, \beta)$ and $b[\alpha, \beta, u(t)]$ are determined by (5) and (15), respectively.

At the first step of the proof, we consider the functionalized equation

$$
u=B(\alpha(u), \beta(u)) u+b[\alpha(u), \beta(u), u],
$$

where $\alpha(u)$ and $\beta(u))$ are continuous functionals chosen as follows

$$
\alpha(u)=\alpha_{0}+\frac{1}{\varepsilon}\left[\left(u, e^{*}\right)-\varepsilon\right], \quad \beta(u)=\beta_{0}+\frac{1}{\varepsilon}\left(u, g^{*}\right) ;
$$

here $\varepsilon>0$ is an auxiliary small parameter.

At the second step, equation (20) is treated by the Newton-Kantorovich method. In order to do it, 20) is represented as (17), where $G(u)=u-B[\alpha(u), \beta(u)] u, W(u)=-b[\alpha(u), \beta(u), u]$.

Space $L_{2}$ can be represented as $L_{2}=H_{0} \oplus H^{0}$, where $H_{0}$ is an eigenspace associated with the semi-simple eigenvalue 1 of multiplicity two of operator $B_{0}: B_{0}=B\left(\alpha_{0}, \beta_{0}\right)$, and $H^{0}$ is the additional invariant for $B_{0}$ subspace. We let $G^{\prime}(\varepsilon e) h=h-\left[\left(h, e^{*}\right) B_{\alpha}^{\prime}\left(\theta_{0}\right) e+\left(h, g^{*}\right) B_{\beta}^{\prime}\left(\theta_{0}\right) e\right]-B_{0} h$ and $u_{0}=\varepsilon e$. Condition (8) of the theorem follows that there exists a bounded operator $\Gamma_{0}=\left[G^{\prime}\left(u_{0}\right)\right]^{-1}: L_{2} \rightarrow L_{2}$. At that, operator $\Gamma_{0}$ is independent of $\varepsilon$. Operator $\Gamma_{0}$ can be calculated by the formula $\Gamma_{0} y=h_{0}+h^{0}$, where

$$
h_{0}=J_{\alpha}(y) e+J_{\beta}(y) g, \quad h^{0}=\left(I-B_{0}\right)^{-1}\left[y+J_{\alpha}(y) B_{\alpha}^{\prime}\left(\theta_{0}\right) e+J_{\beta}(y) B_{\beta}^{\prime}\left(\theta_{0}\right) e\right] .
$$

Here $J_{\alpha}(y)$ and $J_{\beta}(y)$ are calculated by the formula

$$
\left(\begin{array}{c}
J_{\alpha}(y) \\
J_{\beta}(y)
\end{array}\right)=-\left[\begin{array}{cc}
\left(B_{\alpha}^{\prime}\left(\alpha_{0}, \beta_{0}\right) e, e^{*}\right) & \left(B_{\beta}^{\prime}\left(\alpha_{0}, \beta_{0}\right) e, e^{*}\right) \\
\left(B_{\alpha}^{\prime}\left(\alpha_{0}, \beta_{0}\right) e, g^{*}\right) & \left(B_{\beta}^{\prime}\left(\alpha_{0}, \beta_{0}\right) e, g^{*}\right)
\end{array}\right]^{-1}\left(\begin{array}{c}
\left(y, e^{*}\right) \\
\left(y, g^{*}\right)
\end{array}\right) .
$$


Lemma 5. Suppose the hypothesis of Theorem 2. Then for each small $\varepsilon>0$ equation (17) has a solution $u(\varepsilon)$, which can be obtained as the limit of successive approximations

$$
u_{n+1}=u_{n}-\Gamma_{0} G\left(u_{n}\right)-\Gamma_{0} W\left(u_{n}\right), n=0,1,2, \ldots,
$$

where $u_{0}=u_{0}(t)=\varepsilon e(t)$.

It follows from the above constructions that as $\alpha=\alpha[u(\varepsilon)]$ and $\beta=\beta[u(\varepsilon)]$, equation (19) has nonzero solution $u(\varepsilon)$, and hence, $\|u(\varepsilon)-\varepsilon e\|=o(\varepsilon), \alpha[u(\varepsilon)] \rightarrow \alpha_{0}, \beta[u(\varepsilon)] \rightarrow \beta_{0}$ as $\varepsilon \rightarrow 0$. It completes the proof of Theorem 2.

We note that Lemma 5 implies the existence of functions $\alpha(\varepsilon)=\alpha[x(\varepsilon)]$ and $\beta(\varepsilon)=\beta[x(\varepsilon)]$ such that as $\alpha=\alpha(\varepsilon)$ and $\beta=\beta(\varepsilon)$, equation (4) has a non-zero $q T$-periodic solution $x(t, \varepsilon)$ such that $x(0, \varepsilon)=x(\varepsilon)$. The values of functions $\alpha(\varepsilon), \beta(\varepsilon)$, and $x(\varepsilon)$ can be constructed by iterations (21).

Proof of Theorem 3. The proof of this theorem follows the same lines as Theorem 2. The main ingredient is the operator equation

$$
u(t)=B(\theta, T) u(t)+b[\theta, T, u(t)],
$$

where operator $B(\theta, T)$ is determined by identity (11), and nonlinearity $b[\theta, T, u(t)]$ is introduced by the identity

$$
\begin{aligned}
b[\theta, u(t)]= & T \int_{0}^{t}\left(\int_{0}^{\sigma(\theta)}\left[d_{\tau} Q(\theta, \tau)\right] E\left(\frac{\tau}{T}\right) \Phi[\theta, u(s)]\right) d s \\
& +T \int_{0}^{t}\left(F\left(\theta, E\left(\frac{\phi_{1}}{T}\right) u(s), \ldots, E\left(\frac{\phi_{s}}{T}\right) u(s)\right)\right) d s .
\end{aligned}
$$

\section{BIBLIOGRAPHY}

1. R. Bellman, K.L. Cooke. Differential-difference equations. Academic Press, N.Y. (1963).

2. A.D. Myshkis. Linear differential equations with retarded argument. Nauka, Moscow (1972). (in Russian.)

3. J. Hale. Theory of functional differential Equations. Springer, New York (1977).

4. B. Balachandran, T. Kalmar-Nagy, D. Gilsinn. Delay differential equations. Recent advances and new directions. Springer, New York (2009).

5. Yu.S. Kolesov. Justification of the method of quasinormal forms for Hutchinson's equation with a small diffusion coefficient // Izv. RAN. Ser. Matem. 65:4, 111-132 (2001). [Izv. Math. 65:4, 749-768 (2001).]

6. D. Roose, R. Szalai. Continuation and bifurcation analysis of delay differential equations in book "Numerical continuation methods for dynamical systems". Springer, Dordrecht, 359-399 (2007).

7. D. Schley. Bifurcation and stability of periodic solutions of differential equations with statedependent delays // Eur. J. Appl. Math. 14:1, 3-14 (2003).

8. M.I. Kamenskii, Yu.V. Lysakova, P. Nistri. On bifurcation of periodic solutions for functional differential equations of the neutral type with small delay // Avtomatika i Telemekhanika. 12, 41-46 (2008). [Automation and Remote Control. 69:12, 2027-2032 (2008).]

9. A.A. Vyshinskiy, L.S. Ibragimova, S.A. Murtazina, M.G. Yumagulov. An operator method for approximately studying regular bifurcation in multiparameter dynamical systems // Ufimskij Matem. Zhurn. 2:4, 3-26 (2010). (in Russian).

10. M.A. Krasnoselski. Shift operator along trajectories of differential equations. Nauka, Moscow (1966). (in Russian). 
11. B. Hessard, N. Kazarinov, I. Wen. Theory and application of bifurcation of cycle generation. Mir, Moscow (1985). (in Russian.)

12. L.S. Ibragimova, M.G. Yumagulov. Parameter functionalization and its application to the problem of local bifurcations in dynamic systems // Avtomatika i Telemekhanika. 4, 3-12 (2007). [Automation and Remote Control. 68:4, 573-582 (2007).]

13. M.A. Krasnosel'skii, P.P. Zabreiko. Geometric methods of nonlinear analysis. Nauka, Moscow (1975). (in Russian.)

14. M.A. Krasnosel'skii, Vainikko G.M., P.P. Zabreiko, Ya.B. Rutitskii, V.Ya. Stetsenko. Approximative solving of operator equations. Nauka, Moscow (1969). (in Russian.)

Marat Gayazovich Yumagulov,

Bashkir State University,

Zaki Validi, 32,

450074, Ufa, Russia

E-mail: yum_mg@mail.ru

Dina Akhatovna Yakshibaeva,

Sibai branch of Bashkir State University,

Belov str., 21,

453837, Sibai, Russia

E-mail: K_dina_a@mail.ru 